\title{
Interventions for Attention-Deficit Hyperactivity Disorder: A Year in Review
}

\author{
Charlotte Johnston • Joanne L. Park
}

Published online: 7 January 2015

(C) Springer International Publishing Switzerland 2015

\begin{abstract}
Children diagnosed with attention-deficit/hyperactivity disorder (ADHD) experience core symptoms of inattention, hyperactivity, and/or impulsivity, which often result in difficulties in social and psychological functioning and poor outcomes. A wide variety of interventions exist to treat these symptoms and improve associated impairments; some better established than others. Compared to treatments used in isolation, the combination of psychosocial treatment and medication may yield incremental benefits and is highly recommended. Pharmacological interventions are often selected as one component of treatment for ADHD due to convincing evidence of their efficacy in reducing core ADHD symptoms. Psychosocial interventions, such as behavioral parent training (BPT), behavioral classroom interventions, and skills training also have shown benefit for children with ADHD, particularly with regard to improvements in overall functioning. Within the past year, many new studies and reviews have appeared proposing and evaluating new treatments or updates to existing treatments for ADHD. New developments include studies on gene response variability for medication treatment, parent friendship coaching, and cognitive training. This paper provides an up-to-date summary and critical analysis of both new and well-established ADHD interventions.
\end{abstract}

Keywords Attention-deficit/hyperactivity disorder . Pharmacological interventions · Psychosocial interventions . Childhood ADHD

This article is part of the Topical Collection on $A D H D$

C. Johnston $(\bowtie) \cdot$ J. L. Park

Department of Psychology, University of British Columbia,

2136 West Mall, Vancouver, BC V6T 1Z4, Canada

e-mail: cjohnston@psych.ubc.ca

J. L. Park

e-mail: j.park@psych.ubc.ca

\section{Introduction}

Children and adolescents diagnosed with attention-deficit/hyperactivity disorder (ADHD) are at risk for a variety of longlasting educational, vocational, and social impairments that are associated with their core ADHD symptoms of inattention, hyperactivity, and impulsiveness. Treatments to improve these difficulties and outcomes are continually evolving in light of emerging discoveries, such as the specificity in treatment effects and advances in the treatments themselves, as well as new evidence regarding the nature of ADHD. In the past year, these changes included publication of the revised diagnostic criteria in the fifth edition of the Diagnostic and Statistical Manual of Mental Disorders (DSM-5) [1]. The updated criteria for ADHD include the addition of examples that typify ADHD symptoms and behaviors across the life span, the change in required age of onset from before seven-years-old to before 12-years-old, the alternation of subtypes to specifiers, and the movement of ADHD to classification as a neurodevelopmental disorder. There also have been numerous studies published in the past year reporting on existing or new treatments for ADHD.

Based on these recently published studies, this review aims to provide readers with an up-to-date summary and critical analysis of emerging advances in pharmacological and nonpharmacological interventions for ADHD. This review reflects a combination of confirming what is already established in the literature regarding efficacious treatments, as well as highlighting potential new areas of development. First, we examine updates regarding pharmacological interventions. Next, we consider existing and new evidence for psychosocial treatments such as behavioral and skills training interventions. We then comment on the literature describing the combined effects of pharmacological and psychosocial treatments. Last, we address newer interventions that have been proposed but are not well tested or supported, such as cognitive training, 
dietary modifications, and complementary/alternative medicine.

\section{Pharmacological Interventions}

Pharmacological treatments for ADHD are well documented as being mostly safe and efficacious in reducing ADHD symptoms [2]. The most commonly prescribed medications are classified into two broad categories: 1) stimulants, and 2) non-stimulants.

\section{Stimulants}

Stimulants, including both methylphenidate (MPH) and amphetamine (AMP) compounds, are the first-line of pharmacological treatments prescribed to children with ADHD. These compounds act by increasing the availability of dopaminergic and noradrenergic neurotransmitters in the central nervous system (CNS) [3•]. Studies have consistently demonstrated the robust efficacy, an effect size (ES) of .95 to .99 , of stimulants in decreasing ADHD symptoms [4-6]. Other research has documented that stimulants are generally well tolerated in children and are associated with improvements on measures of executive functioning, short-term memory, learning performance, on-task behavior, academic productivity, self-esteem, and with reduced risk-taking behavior [7, 8].

New developments in stimulant medications focus on extended release formulations that allow for more individualization and flexibility of dosages [3•]. For instance, long-acting formulas, designed to cover the entire school day and afterschool hours with a single dose, can be complemented with immediate release preparations for additional coverage in the evenings. Moreover, novel delivery systems allow for more personalized approaches to providing treatment. These systems include solution formulations for children, who have difficulty swallowing pills, and beaded capsule systems that contain different percentages of immediate-release and delayed-release beads to allow for two dosings in one capsule. A transdermal (patch) delivery system is also available.

Despite the ubiquitous use of stimulant drugs to treat ADHD, there is concern for associated adverse events. Several recent studies $[9,10]$ have suggested an increased risk of experiencing a later cardiovascular event when taking stimulants in childhood and children with ADHD on stimulants have significantly higher heart rates than children with ADHD not taking stimulants or children without ADHD [9]. There is also evidence that long-term stimulant use may impact the growth trajectories of children. A systematic analysis of 18 studies found evidence of deficits in height and weight associated with stimulant usage, although the rate of these deficits attenuated over time [11]. In addition, all but two of the studies demonstrated an accelerated growth rate within two years after the discontinuation of medication, which often compensated for the height and weight deficits accrued during medication treatment. Overall, the review suggests that, although growth deficits occur, they are on average small and of limited clinical concern for most children as long as height and weight are carefully monitored. Another common concern is that stimulant treatment may be associated with longer-term risk for substance abuse among children with ADHD. However, research indicates that it is the diagnosis of ADHD, rather than medication treatment, which confers higher risk for substance use disorders [12]. Nevertheless, there may be a subgroup of individuals with ADHD who are prone to abuse stimulants. For instance, Bright [13] found that $14.3 \%$ of participants with ADHD abused prescription stimulants. Because a large percentage of the abused stimulants were short-acting formulations, extended-release formulations are recommended for such individuals $[2,13]$.

Non-stimulants

There is a sizeable percentage ( $30 \%$ ) of children who do not adequately respond to stimulant treatment, or who cannot tolerate side effects [14]. A newer class of "non-stimulant" drugs has been developed that may be beneficial for such children.

Atomoxetine (ATX) ATX acts as a selective noradrenergic reuptake inhibitor. A recent meta-analysis [15] of 25 doubleblind, randomized, placebo-controlled trials $(N=3928)$ found that ATX was superior to placebo on a number of outcomes, including decreases in inattentive and hyperactive ADHD symptom scores $(\mathrm{ES}=.59$ to .67$)$, decreases in oppositional behaviors $(\mathrm{ES}=.33)$, increases in the child's quality of life $(\mathrm{ES}=.39)$, and fewer inefficacy-related discontinuations of medication. However, the meta-analysis also identified a significantly higher rate of psychiatric adverse events (e.g., suicidal ideation) for ATX compared to placebo. The demonstrated positive effect sizes for symptom reduction, although favoring ATX over placebo, suggest that the primary efficacy of ATX for core ADHD symptoms may be lower than the effect size commonly reported with stimulants. However, very few head-to-head trials comparing stimulants and ATX exist; ATX was inferior to MPH in one study [16], but not the other [17]. In these studies, ATX demonstrated similar side effects to stimulants, most of which were mild-to-moderate in severity. Similarly, Bushe and Savill [18] conducted a metaanalysis that compared suicide-related events between ATX and MPH treatments and found that both drugs had low overall suicide risk with no differences in suicide-related events between the two medication classes. From these studies, we can conclude that ATX is superior to placebo in reducing ADHD symptomatology, and is usually well tolerated. Future research is needed to compare ATX with other well- 
established medications (e.g., MPH), as well as address the findings on adverse psychiatric events.

Alpha-2-Adrenergic (A2A) Agonists A2A agonists, such as clonidine and guanfacine, act by binding to $\alpha-2$ receptors in the prefrontal cortex. Commonly prescribed for hypertension, extended release formulations of A2As have been recently approved by the FDA in the United States to treat pediatric ADHD [3•]. Hirota and colleagues [19] performed a metaanalysis of 12 studies $(N=2276)$ that demonstrated robust efficacy for A2A monotherapy (ES=.56-.59), and to a lesser degree, as an add-on to stimulant treatment $(\mathrm{ES}=.32-.36)$ in reducing hyperactive/impulsive and inattentive symptoms. Because in the studies measuring the efficacy of A2A as an add-on treatment, participants were already taking stimulants at baseline, the smaller effect sizes for $\mathrm{A} 2 \mathrm{~A}$ as a combination treatment are expected. Compared to placebo or no treatment, A2A treatment was associated with fatigue, somnolence, sedation, and decreases in blood pressure and heart rate. When comparing effect sizes across different medications, A2A monotherapy appears to have 30-60\% lower efficacy than stimulants, and may be comparable to the efficacy of ATX. Despite being less efficacious overall, the incremental effect associated with the addition of A2A to existing medication treatment suggests its utility as a useful add-on for children who have an insufficient response to stimulants. Future research is needed to more directly and fully compare the benefits and potential risks of A2A treatments with other drug treatments such as ATX and MPH.

\section{New and Future Directions}

Given the evidence that a sizable minority of children treated with stimulants do not respond, or do not tolerate the medication [14], and that certain groups of children respond preferentially to different medications [20], research has moved to a focus on the gene-related predictors of this response variability. For example, Kambeitz and colleagues [21] studied the moderating effect of the SLC6A3 (a gene that codes for the dopamine transporter) variable number tandem repeat (VNTR) polymorphism on responses to MPH treatment in a meta-analysis. Disappointingly, the results from 16 studies $(N=1572)$ indicated no effect of variations of the SLC6A3 VNTR on children's response to MPH. However, these results do not preclude the possibility that other variations in this gene, other genes, or combinations of genes may serve as moderators of pharmacological treatment response, and future studies in this area are warranted.

Overall, both stimulants and non-stimulants have been established as efficacious and safe treatments for pediatric ADHD. However, a review of this literature does raise issues regarding the extent to which studies are funded by pharmaceutical companies, or study authors report other conflicts of interest $[4,16,18]$. The validity of future medication trials would be enhanced if such conflicts of interest could be avoided, and it would be useful in meta-analyses to consider whether such conflicts moderate treatment effects.

\section{Psychosocial Interventions}

In this section, we consider recent evidence regarding a range of psychosocial treatments for childhood ADHD. Several recent reviews summarize the effects of these treatments, however, these reviews have differed in methodologies and, therefore, in their conclusions. We highlight some of these differences in order to parse the most reasonable conclusions that can be reached regarding these treatments. In 2013, Sonuga-Barke and colleagues [22] published a review suggesting limited evidence supporting most psychosocial treatments and this stands in contrast to other reviews such as those by Evans and colleagues [23•], Daley and colleagues [24], and by Fabiano and colleagues [25], which have argued that at least some forms of psychosocial treatment are supported by strong evidence of efficacy as well as real world effectiveness. These differences in conclusions seem attributable to multiple factors in the conduct of the reviews. The Sonuga-Barke et al., review [22] included only randomized controlled trials and restricted outcomes to measures of ADHD symptoms. In addition, their negative conclusions reflected only outcomes assessed using blinded assessments (e.g., observations, ratings by teachers who were unaware of home treatments), which although more objective than ratings by those involved in the treatment, may not have adequately captured changes in the child's behavior. In contrast, other reviews [23•, 24, 25] have defined outcomes to include impairments and other problems related to ADHD (e.g., oppositional behavior), included both single case and group research designs, and/or examined both blinded and non-blinded evaluations of the treatment. In sum, aggregating across the multiple reviews, we believe the best supported conclusion is that there is firm evidence for the efficacy of several types of psychosocial treatments, including behavioral parent training, classroom management and other school interventions, and some types of skills training, particularly when aspects of the child's functioning beyond just ADHD symptoms are the targeted outcomes. However, as we note below, other types of psychosocial treatments have very limited evidence of positive effects and further study is needed to fully understand the effectiveness parameters of all psychosocial interventions.

Behavioral and Skills Training Interventions

Behavioral Parent Training (BPT) BPT is arguably the most well-studied and supported psychosocial treatment for ADHD 
$[23 \cdot, 24]$. BPT teaches parents more systematic and effective ways of coping with their child's ADHD symptoms and associated disruptive behavior problems, such as building a positive parent-child relationship, encouraging appropriate child behavior with incentive systems, and implementing appropriate and consistent consequences for child misbehavior. Previous reviews have concluded that BPT is a wellestablished treatment for children with ADHD (ES=.47-.70) $[24,26,27]$. Six additional, recent studies evaluating BPT have all documented significant benefits on parent ratings of child symptoms and impairment compared to control conditions (waitlist or routine care) [23•]. However, traditional BPT programs often have disappointing rates of engagement and retention, perhaps due to low parent acceptance of the rationale supporting BPT, or the high degree of parent involvement required by the treatment [28]. Therefore, research is now moving to identify factors that may improve parental engagement and retention in BPT. Five of the six studies mentioned above evaluated enhancements to the structure of BPT in order to target individuals not normally well served by traditional BPT. For instance, Fabiano and colleagues [29] sought to increase father involvement by creating a program that delivered behavior management skills training in the context of fathers coaching their children to play soccer. The program proved similar to traditional BPT on most outcomes and fathers in the enhanced program reported significantly greater improvement in child behavior. Other enhanced BPT programs include extended sessions for single mothers [30], telephone-based BPT [31], group BPT [32], and programs targeting specific difficult populations (e.g., depressed mothers [33]). Overall, these studies found that the enhanced programs are as effective as traditional BPT (although there is limited evidence of incremental benefit) and the hope is that they will prove valuable as a means of increasing participant engagement for traditionally underserved families (e.g., single mothers, low SES families).

Other new developments in BPT include the increased use of technology to retain families in treatment. For example, Jones and colleagues [28] are investigating the use of smartphone technology. This intervention includes mid-week videoconferencing calls with the therapist (using two-way phone cameras), and videotaping of parent-child interactions at home for the therapist to review and provide feedback on during sessions. Preliminary results indicate that this technology-enhanced BPT is more effective than telephone consultation only [28]. Such technology offers considerable potential that is only beginning to be explored in psychosocial interventions. Future directions in BPT interventions may rest with technologies such as podcasts, smartphone applications, and virtual reality to further enhance and improve the acceptability and generalizability of treatment effects.
Parental Friendship Coaching Although BPT programs are effective in reducing noncompliance among children with ADHD, improvements in children's social functioning outside of the home are not targeted or may not persist once treatment is discontinued [6]. Traditional social skills training (SST) programs were developed to address these limitations. However, evidence suggests that such SST programs often do not result in generalization of improved social behaviors outside of the treatment group settings [34, 35]. It has been argued that this failure rests in part on a key, and likely incorrect, assumption of traditional SST programs that children with ADHD have a knowledge deficit, rather than a performance deficit in displaying positive social skills. Traditional SST programs also often ignore the contextual factors surrounding the social difficulties of children with ADHD. To address these issues, Mikami and colleagues [36] developed a novel program to train parents to become "friendship coaches" for their children with ADHD. Their rationale was that parents shape their children's social context and are likely to be present when their children are interacting with peers, and therefore, are able to enhance performance by reminding their children to use positive social skills "in the moment." Post-test results from a pilot study of this intervention indicated that parents who received Parental Friendship Coaching (PFC) had children with better social behaviors (parent-report) and who were more accepted and less rejected by peers (teacher-report) compared to the control group [36]. At 1-month follow-up, the PFC group reported significant continued improvements in children's friendship relative to the control group. Based on this study, we offer a tentative conclusion that PFC may address the limitations of traditional unsuccessful SST programs, and offers a promising new social skills intervention. Replications of these findings and longer-term follow-ups, as well as comparisons with established treatments, such as BPT, are needed moving forward.

Classroom Interventions In addition to treatments focused on improving children's functioning in the home context and with peers, school interventions targeting the classroom behavior and academic performance of children with ADHD also are well-established and supported [23•, 37•, 38], and confer moderate to large effects on both behavior $(\mathrm{ES}=.18-.72)$ and academic outcomes $(\mathrm{ES}=.42-.43)$. Classroom behavior management, including proactive strategies such as establishing clear rules and using visual reminders as well as reactive strategies such as the use of positive and negative consequences, form the bulwark of these treatments with useful additional components such as daily report cards and the inclusion of training in self-regulation skills. Research also supports a variety of consultation models used to assist classroom teachers in developing and consistently implementing these interventions [38]. Beyond the elementary school level, there are greater challenges to school 
interventions (e.g., adolescents often have multiple teachers, greater independence is expected), but encouragingly, there is evidence supporting the use of multi-component psychosocial programs to improve school functioning even at this age [39]. Similarly, recent studies support the usefulness of combining school interventions with those targeting parent or child skills training [40, 41].

A new development in classroom interventions focuses on improving peer regard, and as a result, reducing social impairment in children with ADHD. Peers frequently hold negative attitudes and perceptions, and behave in a negative manner toward children with ADHD, often within minutes of their first meeting [42]. The Making Socially Accepting Inclusive Classrooms (MOSAIC) intervention includes traditional behavioral management techniques and attempts to improve the social lives of children with ADHD by addressing peers' negative impressions [43]. This is done by training teachers in three main principles: 1) to adjust their own responses to a child with ADHD to be more tolerant and positive, 2) to train the peer group to refrain from excluding children with ADHD, and 3) to draw attention to positive characteristics of children with ADHD in order to dismantle peers' negative impressions. In a pilot randomized trial, Mikami and colleagues [43] compared MOSAIC to a traditional classroom behavioral management program and found that problem behaviors of children with ADHD were equally controlled in both groups. Moreover, children receiving the MOSAIC treatment had improved social standing, more reciprocated friendships, and received more positive messages from peers compared to children in the traditional program. These findings are promising and bear replication over a longer time period. As this pilot study was conducted within the context of a summer treatment program, future studies will need to address the usability and efficacy of the MOSAIC intervention in general education classrooms during the school year.

Skills Training As noted above, previous interventions working directly with children with ADHD to teach social skills or to address intra-psychic difficulties yielded disappointing results $[34,35]$. In contrast, recent evidence has supported treatments that work directly with the child to target compensatory skills, such as organizational skills, to improve functioning in areas impaired by ADHD (ES=.42-2.77) [44, 45]. Focusing on the school context, these interventions help children and youth develop necessary organizational skills (e.g., using checklists, breaking tasks into steps) and combine this training with rewards for the child's use of these skills. Randomized controlled trials offer evidence that such organizational training is not only better than wait list control conditions, but is often better than contingency-based treatments (e.g., rewards) used alone $[44,45]$.
Cognitive/Executive Functioning Training Treatments

A relatively recent addition to the ADHD treatment scene is a group of treatments focused on enhancing cognitive or executive functioning, typically using a facilitative intervention approach with progressively more difficult computer tasks used to improve what are presumed to be core executive functioning deficits in children with ADHD. These treatments have garnered wide interest, perhaps in part due to the limitations in improvement that are apparent for many children with ADHD, even when well-established pharmacological and psychosocial treatments are combined $[12,46]$. Several meta-analyses and reviews of these cognitive/executive functioning training programs have appeared within the recent literature, including studies conducted with both clinical and typical samples $[23 \cdot, 47,48]$. These reviews show a consensus of supportive evidence for the immediate treatment effects of these cognitive interventions on tasks that are similar to the trained skill or on non-blinded evaluations of ADHD symptoms. However, little evidence supports sustained effects of these treatments or generalization to other skills or to academic or behavioral functioning [23•, 47]. In addition, a number of limitations to these treatments have been noted, including concerns that they do not necessarily target the executive functions they propose to teach (e.g., using tasks that train visual memory storage rather than the purported focus on working memory). With a few exceptions, well-controlled randomized trials evaluating these treatments with appropriate samples and measures of a range of aspects of functioning conducted at both post-treatment and over time are not available and, as such, the potential of these cognitive training programs, as well as other cognitive interventions such as mindfulness-based training or neurofeedback training, remains unsubstantiated.

\section{Combined Pharmacological and Psychosocial Treatments}

Combining pharmacological and psychosocial treatments, such as behavioral parent training or classroom management programs, is supported based on both evidence of the effects of each of the treatments used in isolation, as well as the clinical reality that the use of single treatments is seldom sufficient to manage the totality of impairments experienced by children with ADHD [49]. Meta-analyses and reviews $[23 \cdot, 25,38,50]$, as well as longer-term outcome data from the large multi-site treatment of ADHD study (MTA) [12], all provide evidence that treatments that combine medication with behavioral treatments, both in the home and school contexts, are best for managing behavioral and social problems and impairments, and for preventing longer-term difficulties such as substance use or antisocial behavior among 
children with ADHD. The influence of these treatments on academic problems is less well documented [50].

Unfortunately, combined treatments are often both expensive and time-consuming, and may be perceived as of limited feasibility. Countering these difficulties, recent studies show that using medication and behavioral treatments in combination permits positive effects to be achieved at lower doses of both treatments (e.g., less medication and less intensive/ extensive behavioral treatment) [51]. Thus, we believe that combined pharmacological and behavioral interventions remain the best treatment options for most children with ADHD and efforts are needed to refine methods for individualizing the best possible pairing or sequencing of these interventions so as to maximize their clinical utility.

\section{Other Non-pharmacological Treatments}

As the last category of treatments for ADHD that we review, we briefly consider evidence for other non-pharmacological treatments such as those of a dietary or complementary medicine nature. In reviewing these treatments, Hurt and Arnold [52] highlight the need for evaluations of these interventions to be based not only on consideration of the very limited empirical evidence available, but also to include attention to the extent of risk and cost associated with the unproven treatment. Meta-analyses have suggested that elimination diets and diets high in essential fatty acids may have small effects on the behavior of children with ADHD $(\mathrm{ES}=.29)$, although these benefits do not appear to be limited to individuals with ADHD and do not occur for sugar elimination [22, 53]. There is limited to no support for a range of other alternative treatments such as dietary supplements, yoga, meditation, massage, homeopathy, acupuncture, or the use of devices such as weighted vests [54], with few existing studies and fewer still well-controlled studies. One possible exception to this list is the use of physical exercise, which in preliminary studies has shown some potential benefits as a treatment for $\mathrm{ADHD}$ and would rank low on perceived risks or costs [55].

\section{Conclusion}

As this brief review indicates, a number of pharmacological and psychosocial interventions and their combinations exist which have demonstrated positive effects on the functioning of children with ADHD. In addition, these relatively wellestablished treatments are being supplemented by a number of new, innovative treatment approaches that offer considerable potential to broaden the range of treatment options available and to enhance both the breadth of coverage and intensity of effects obtained. At the same time, the effects of these treatments on the long-term outcomes of individuals with ADHD remain far from optimal [12] and we encourage continued research to develop and identify even more effective interventions.

In closing, we touch on two issues related to the dissemination and uptake of interventions that may prove important as the field searches for methods to enhance and expand effective treatments for ADHD. The first of these is a need to give greater consideration to parent and youth treatment preferences given the critical role that these attitudes play in treatment engagement [56]. Approaching the treatment of ADHD in a manner that respects and addresses these attitudes and seeks to collaborate with children and families may offer opportunities to enhance the utility of existing treatments. The second issue is the necessary work needed to improve the consistent implementation and monitoring of evidence-based treatments in order to maximize outcomes. One example of such work is the online intervention developed by Epstein and colleagues [57] that facilitates clinical practice aspects such as the assessment of ADHD, monitoring of treatment response, and collaborative communication among treatment providers, parents, and school personnel. We believe that efforts such as these will promote greater effectiveness and clinical impact of the existing proven treatments for ADHD.

\section{Compliance with Ethics Guidelines}

Conflict of Interest Charlotte Johnston and Joanne L. Park declare that they have no conflict of interest.

Human and Animal Rights and Informed Consent This article does not contain any studies with human or animal subjects performed by any of the authors.

\section{References}

Papers of particular interest, published recently, have been highlighted as:

- Of importance

1. American Psychiatric Association. Diagnostic and statistical manual of mental disorders. 5th ed. Arlington: American Psychiatric Association; 2013.

2. Cortese S, Holtmann M, Banaschewski T, et al. Practitioner review: current best practice in the management of adverse events during treatment with ADHD medications in children and adolescents. J Child Psychol Psychiatry. 2013. doi:10. 1111/jcpp. 12036.

3. Connor DF. Stimulant and nonstimulant medications for childhood ADHD. In: Barkley RA, editor. Attention-deficit hyperactivity disorder: a handbook for diagnosis and treatment. 4th ed. New York: Guilford; in press. This chapter provides a recent and comprehensive review of pharmacological interventions for $A D H D$ in children. 
4. Faraone SV, Buitelaar J. Comparing the efficacy of stimulants for ADHD in children and adolescents using meta-analysis. Eur Child Adolesc Psychiatry. 2010. doi:10.1007/s00787-009-0054-3.

5. MTA Cooperative Group. A 14-month randomized clinical trial of treatment strategies for attention-deficit/hyperactivity disorder. Arch Gen Psychiatry. 1999. doi:10.1001/archpsyc.56.12.1073.

6. MTA Cooperative Group. National institute of mental health multimodal treatment study of ADHD follow-up: 24-month outcomes of treatment strategies for attention-deficit/hyperactivity disorder. Pediatrics. 2004;113:754-61.

7. DeVito EE, Blackwell AD, Kent L, et al. The effects of methylphenidate on decision making in attention-deficit/hyperactivity disorder. Biol Psychiatry. 2008. doi:10.1016/j.biopsych.2008.04.017.

8. Prasad V, Brogan E, Mulvaney C, et al. How effective are drug treatments for children with ADHD at improving on-task behaviour and academic achievement in the school classroom? A systematic review and meta-analysis. Eur Child Adolesc Psychiatry. 2012. doi: 10.1007/s00787-012-0346-x.

9. Hailpern SM, Egan BM, Lewis KD, et al. Blood pressure, heart rate, and CNS stimulant medication use in children with and without ADHD: analysis of NHANES data. Front Pediatr. 2014. doi:10. 3389/fped.2014.00100.

10. Dalsgaard S, Kvist AP, Leckman JF, Nielsen HS, Simonsen M. Cardiovascular safety of stimulants in children with attention-deficit/hyperactivity disorder: a nationwide prospective cohort study. J Child Adolesc Psychopharmacol. 2014. doi:10.1089/cap.2014. 0020 .

11. Faraone SV, Biederman J, Morley CP, Spencer TJ. Effect of stimulants on height and weight: a review of the literature. J Am Acad Child Adolesc Psychiatry. 2008. doi:10.1097/CHI. ObO13e31817eOea7.

12. Molina BSG, Hinshaw SP, Arnold LE, et al. Adolescent substance use in the multimodal treatment study of attention-deficit/hyperactivity disorder (ADHD) (MTA) as a function of childhood ADHD, random assignment to childhood treatments, and subsequent medication. J Am Acad Child Adolesc Psychiatry. 2013. doi:10.1016/j. jaac.2012.12.014.

13. Bright GM. Abuse of medications employed for the treatment of ADHD: results from a large-scale community survey. Medscape $\mathrm{J}$ Med. 2008;10:111.

14. Pliszka S. Practice parameter for the assessment and treatment of children and adolescents with attention-deficit/hyperactivity disorder. J Am Acad Child Adolesc Psychiatry. 2007. doi:10.1097/chi. 0b013e318054e724.

15. Schwartz S, Correll CU. Efficacy and safety of atomoxetine in children and adolescents with attention-deficithyperactivity disorder: results from a comprehensive meta-analysis and metaregression. J Am Acad Child Adolesc Psychiatry. 2014. doi: 10.1016/j.jaac.2013.11.005.

16. Newcorn JH, Kratochvil CJ, Allen AJ, et al. Atomoxetine and osmotically released methylphenidate for the treatment of attention deficit hyperactivity disorder: acute comparison and differential response. Am J Psychiatry. 2008. doi:10.1176/appi.ajp.2007. 05091676.

17. Wang Y, Zheng Y, Du Y, et al. Atomoxetine versus methylphenidate in paediatric outpatients with attention deficit hyperactivity disorder: a randomized, double-blind comparison trial. Aust N Z J Psychiatr. 2009. doi:10.1080/00048670601057767.

18. Bushe CJ, Savill NC. Systematic review of atomoxetine data in childhood and adolescent attention-deficit hyperactivity disorder 2009-2011: focus on clinical efficacy and safety. J Psychopharmacol. 2014. doi:10.1177/0269881113478475.

19. Hirota T, Schwartz S, Correll CU. Alpha-2 agonists for attentiondeficit/hyperactivity disorder in youth: a systematic review and meta-analysis of monotherapy and add-on trials to stimulant therapy. J Am Acad Child Adolesc Psychiatry. 2014. doi:10.1016/ j.jaac.2013.11.009.

20. Greenhill LL, Abikoff HB, Arnold LE, Cantwell DP. Medication treatment strategies in the MTA study: relevance to clinicians and researchers. J Am Acad Child Adolesc Psychiatry. 1996. doi:10. 1097/00004583-199610000-00017.

21. Kambeitz J, Romanos M, Ettinger U. Meta-analysis of the association between dopamine transporter genotype and response to methylphenidate treatment in ADHD. Pharmacogenomics J. 2014. doi:10.1038/tpj.2013.9.

22. Sonuga-Barke E, Brandeis D, Cortese S, et al. Nonpharmacological interventions for ADHD: systematic review and meta-analyses of randomized controlled trials of dietary and psychological treatments. Am J Psychiatry. 2013;170:275-89.

23. Evans SW, Owens JS, Bunford N. Evidence-based psychosocial treatments for children and adolescents with attention-deficit/hyperactivity disorder. J Clin Child Adolesc Psychol. 2013. doi:10.1080/ 15374416.2013.850700. This article summarizes recent evidence supporting psychosocial interventions for ADHD.

24. Daley D, Van der Oord S, Ferrin M, et al. Behavioral interventions in attention-deficit/hyperactivity disorder: a meta-analysis of randomized controlled trials across multiple outcome domains. J Am Acad Child Adolesc Psychiatry. 2014. doi:10.1016/j.jaac.2014.05. 013.

25. Fabiano GA, Pelham Jr WE, Coles EK, et al. A meta-analysis of behavioral treatments for attention-deficit/hyperactivity disorder. Clin Psychol Rev. 2009. doi:10.1016/j.cpr.2008.11.001.

26. Pelham Jr WE, Fabiano GA. Evidence-based psychosocial treatments for attention-deficit/hyperactivity disorder. J Clin Child Adolesc Psychol. 2008. doi:10.1080/15374410701818681.

27. Pelham Jr WE, Wheeler T, Chronis A. Empirically supported psychosocial treatments for attention deficit hyperactivity disorder. J Clin Child Psychol. 1998;27:190-205.

28. Jones DJ, Forehand R, Cuellar J, et al. Harnessing innovative technologies to advance children's mental health: behavioral parent training as an example. Clin Psychol Rev. 2013. doi:10.1016/j.cpr. 2012.11.003.

29. Fabiano GA, Chacko A, Pelham Jr WE, et al. A comparison of behavioral parent training programs for fathers of children with attention-deficit/hyperactivity disorder. Behav Ther. 2009. doi:10. 1016/j.beth.2008.05.002.

30. Chacko A, Wymbs BT, Wymbs FA, et al. Enhancing traditional behavioral parent training for single mothers of children with ADHD. J Clin Child Adolesc Psychol. 2009. doi:10.1080/ 15374410802698388.

31. McGrath PJ, Lingley-Pottie P, Thurston C, et al. Telephone-based mental health interventions for child disruptive behavior or anxiety disorders: randomized trials and overall analysis. J Am Acad Child Adolesc Psychiatry. 2011. doi:10.1016/j.jaac.2011.07.013.

32. Van Den Hoofdakker BJ, Van Der Veen-Mulders L, Sytema S, et al. Effectiveness of behavioral parent training for children with ADHD in routine clinical practice: a randomized controlled study. J Am Acad Child Adolesc Psychiatry. 2007. doi:10.1097/chi. 0b013e3181354bc2.

33. Chronis-Tuscano A, Clarke TL, O'Brien KA, et al. Development and preliminary evaluation of an integrated treatment targeting parenting and depressive symptoms in mothers of children with attention-deficit/hyperactivity disorder. J Consult Clin Psychol. 2013. doi:10.1037/a0032112.

34. Abikoff H, Hechtman L, Klein RG, Gallagher R. Social functioning in children with ADHD treated with long-term methylphenidate and multimodal psychosocial treatment. J Am Acad Child Adolesc Psychiatry. 2004. doi:10.1097/01.chi.0000128797.91601.1a.

35. Antshel KM, Remer R. Social skills training in children with attention deficit hyperactivity disorder: a randomized-controlled 
clinical trial. J Clin Child Adolesc Psychol. 2003. doi:10.1207/ S15374424JCCP3201_14.

36. Mikami AY, Lerner MD, Griggs MS, et al. Parental influence on children with attention-deficit/hyperactivity disorder: II. Results of a pilot intervention training parents as friendship coaches for children. J Abnorm Child Psychol. 2010. doi:10.1007/s10802-0109403-4.

37. DuPaul GJ, Eckert TL, Vilardo B. The effects of school-based interventions for attention deficit hyperactivity disorder: a metaanalysis 1996-2010. School Psychol Rev. 2012;26:5-27. This review summarizes evidence supporting school-based interventions for $A D H D$.

38. Pfiffner LJ, DuPaul GJ. Treatment of ADHD in school settings. In: Barkley RA, editor. Attention-deficit hyperactivity disorder: a handbook for diagnosis and treatment. 4th ed. New York: Guilford; in press.

39. Evans SW, Schultz BK, DeMars CE, Davis H. Effectiveness of the challenging horizons after-school program for young adolescents with ADHD. Behav Ther. 2011. doi:10.1016/j.beth.2010.11.008.

40. Pfiffner LJ, Hinshaw SP, Owens E, et al. A two-site randomized clinical trial of integrated psychosocial treatment for ADHDinattentive type. J Consult Clin Psychol. 2014. doi:10.1037/ a0036887.

41. Power TJ, Mautone JA, Soffer SL, et al. A family-school intervention for children with ADHD: results of a randomized clinical trial. J Consult Clin Psychol. 2012. doi:10.1037/a0028188.

42. Erhardt D, Hinshaw SP. Initial sociometric impressions of attentiondeficit hyperactivity disorder and comparison boys: predictions from social behaviors and from nonbehavioral variables. J Consult Clin Psychol. 1994. doi:10.1037/0022-006X.62.4.833.

43. Mikami AY, Griggs MS, Lerner MD, et al. A randomized trial of a classroom intervention to increase peers' social inclusion of children with attention-deficit/hyperactivity disorder. J Consult Clin Psychol. 2013. doi:10.1037/a0029654.

44. Abikoff H, Gallagher R, Wells KC, et al. Remediating organizational functioning in children with ADHD: immediate and longterm effects from a randomized controlled trial. J Consult Clin Psychol. 2013. doi:10.1037/a0029648.

45. Langberg JM, Epstein JN, Becker SP, Girio-Herrera E, Vaughn AJ. Evaluation of the homework, organization, and planning skills (HOPS) intervention for middle school students with attention deficit hyperactivity disorder as implemented by school mental health providers. School Psychol Rev. 2012;41:342-64.
46. Jensen PS, Arnold LE, Swanson JM, et al. 3-year follow-up of the NIMH MTA study. J Am Acad Child Adolesc Psychiatry. 2007. doi:10.1097/CHI.0b013e3180686d48.

47. Rapport MD, Orban SA, Kofler MJ, et al. Executive function training for children with ADHD. In: Barkley RA, editor. Attention-deficit hyperactivity disorder: a handbook for diagnosis and treatment (4th ed). New York: Guilford; in press.

48. Melby-Lervåg M, Hulme C. Is working memory training effective? A meta-analytic review. Dev Psychol. 2013. doi:10.1037/ a0028228.

49. Smith BH, Shapiro CJ. Combined treatments. In: Barkley RA, editor. Attention-deficit hyperactivity disorder: a handbook for diagnosis and treatment. 4th ed. New York: Guilford; in press.

50. Van der Oord S, Prins PJM, Oosterlaan J, Emmelkamp PMG. Efficacy of methylphenidate, psychosocial treatments and their combination in school-aged children with ADHD: a meta-analysis. Clin Psychol Rev. 2008. doi:10.1016/j.cpr.2007.10.007.

51. Pelham WE, Burrows-MacLean L, Gnagy EM, et al. A doseranging study of behavioral and pharmacological treatment in social settings for children with ADHD. J Abnorm Child Psychol. 2014. doi:10.1007/s10802-013-9843-8.

52. Hurt E, Arnold LE. Dietary management of ADHD. In: Barkley RA, editor. Attention-deficit hyperactivity disorder: a handbook for diagnosis and treatment. 4th ed. New York: Guilford; in press.

53. Nigg JT, Lewis K, Edinger T, Falk M. Meta-analysis of attentiondeficit/hyperactivity disorder or attention-deficit/hyperactivity disorder symptoms, restriction diet, and synthetic food color additives. J Am Acad Child Adolesc Psychiatry. 2012. doi:10.1016/j.jaac. 2011.10.015.

54. Bader A, Adesman A. Complementary and alternative medicine for ADHD. In: Barkley RA, editor. Attention-deficit hyperactivity disorder: a handbook for diagnosis and treatment. 4th ed. New York: Guilford; in press.

55. Rommel AS, Halperin JM, Mill J, Asherson P, Kuntsi J. Protection from genetic diathesis in attention-deficit/hyperactivity disorder: possible complementary roles of exercise. J Am Acad Child Adolesc Psychiatry. 2013;52:900-10.

56. Fiks AG, Mayne S, DeBartolo E, et al. Parental preferences and goals regarding ADHD treatment. Pediatrics. 2013. doi:10.1542/ peds. 2013-0152.

57. Epstein JN, Langberg JM, Lichtenstein PK, et al. The myADHDportal.Com Improvement Program: an innovative quality improvement intervention for improving the quality of ADHD care among community-based pediatricians. Clin Pract Pediatr Psychol. 2013. doi:10.1037/cpp0000004. 